Great activity reigned in the lobby of the largest hotel in Split as numerrous delegates converged on it, some to participate in a conference on European historical cities and others to join the EPS Council Session on 19 and 20 October 1971. 'History' and 'Physics ' were safely separated and channelled into their respective conference rooms. The meetings proceeded smoothly and agreeably thanks to much preparatory work done by Professor N. Cindro, EPS Vice-Secretary, and his aids. Not only did he take good care of the organization but he also acted as host at a pleasant al fresco lunch under palms at his family home outside Split where Venetians and Turks once faced each other across their common frontier.

At the opening session the EPS Council was greeted by Mr. V. Mesko representing the Minister of Science of the Republic of Croatia, who emphasized the important role of the EPS in furthering international relations between physicists in Europe, and the tasks it could perform in helping students in smaller countries and the younger scientists. In thanking him the President confirmed that these were important aims of the Society.

Following the President's review of the activities of the Advisory Committees, W. Martienssen gave an account of the preparations and programme arrangements for the Wiesbaden General Conference. A number of programme modifications were suggested and an appeal was made that

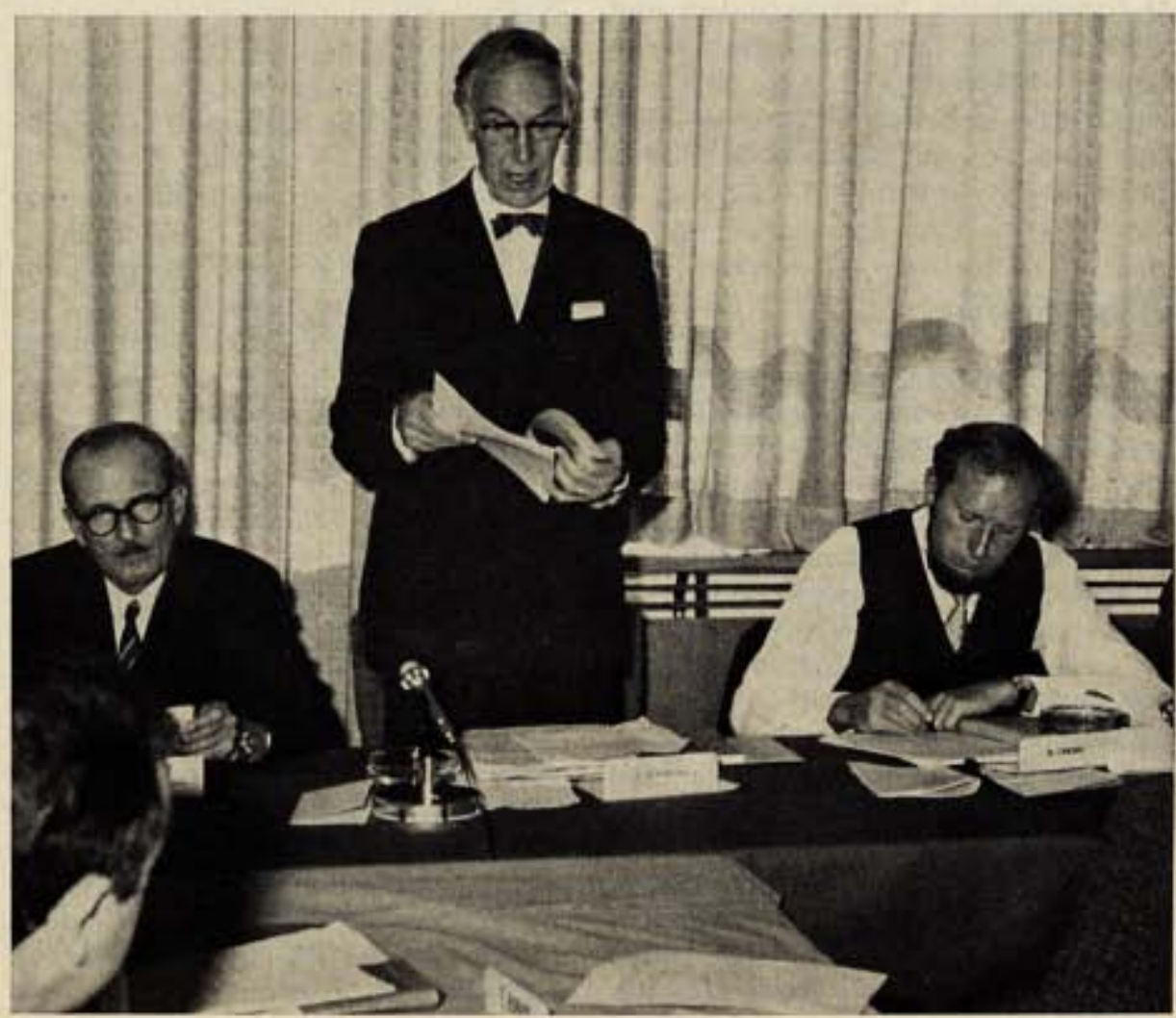

young people should be helped to attend the Conference which, notwithstanding some financial restraints, promises to be an outstanding event in next year's calendar of scientific gatherings.

Financial aspects came again to the fore in the subsequent lively discussion of a report by the Special Administration Study Committee. The operation of the Central Office had been examined so that procedural changes could be suggested which would lead to a reduction in administrative costs. The growth in membership and in the manifold activities of the Society inevitably exerts a mounting pressure on the Main Secretariat. One of the problems is to decide on the priorities regarding the tasks to be performed there within the limits set by the present financial situation. Savings could result from a simplification of the rules for individual membership and in asking the National Societies to take over a larger share of the responsibilities for admission of Individual Ordinary Members, fee collection and distribution of papers. The operation of the Advisory Committees may also

\section{At the Council Session in Split, Yugoslavia}

\author{
W. S. Newman
}

\section{.} cutive terms.

The opportunity for a closer examination of the financial situation came with the presentation of the Auditors' report on the year 1970 and the presentation by the Treasurer of the budgets for 1971 and 1972. After two years in the "red" it was good to find income exceeding expenditure: in effect, for 1971, the provisional positive balance of Sw. Frs 53051 slightly exceeds the figure forecast. Various items of expenditure were discussed in detail and the wish was expressed to increase the contributions to the Divisions. In the budget adopted by Council for 1972 a positive balance of Sw. Frs 22613 is forecast. It remains to be seen whether the target figures for membership and donations can be achieved and what financial consequences will result from the proposed reorganization measures.

After so much discussion of weighty administrative and financial problems it was with some relief that Delegates 
turned their attention towards the more congenial aspects of physics exemplified by the activities of the Specialized Divisions. Clearly some very useful work is being done there as indicated by the various divisional reports presented to Council.

It was with great pleasure that Council accepted the application of the Polish Physical Society to join as from 1 January 1972. There are now very few countries in Europe where one or more national groups of physicists are not members of the EPS, and the Executive Committee will be investigating the possibilities the missing national societies have of joining the EPS. With the recent batch of applications for Individual Ordinary Membership accepted by Council the total now stands at 1901 individual members. Allowing for applications in the pipeline the figure should be close to the 2000 mark at the end of the year and with a determined effort the 1971 budget target of 2500 (see graph) could well be reached by the time Council meets in Oslo on 14 and 15 March 1972.

Council business over, delegates assembled once more, this time not in a conference room but in the heart of the old town to be expertly guided by Professor Cindro through its floodlit squares and dark alleys. The interests of 'History' and 'Physics' had merged in the end.

\section{Polymer}

the science and technology of polymers and biopolymers.

Polymer is the international medium for the publication of original papers on the chemistry, physics and application of polymer research and on other disciplines which contribute to the development of polymer science. Polymer is published monthly to ensure the swift appearance in print of papers accepted for publication. All papers are scrupulously refereed and the journal is guided by an editorial board of scientists whose activities cover all aspects of polymer research. One-year subscription (twelve issues) £ 18.50 (\$ 46.25) from IPC Science and Technology Press, 32 High Street, Guildford, Surrey, England.

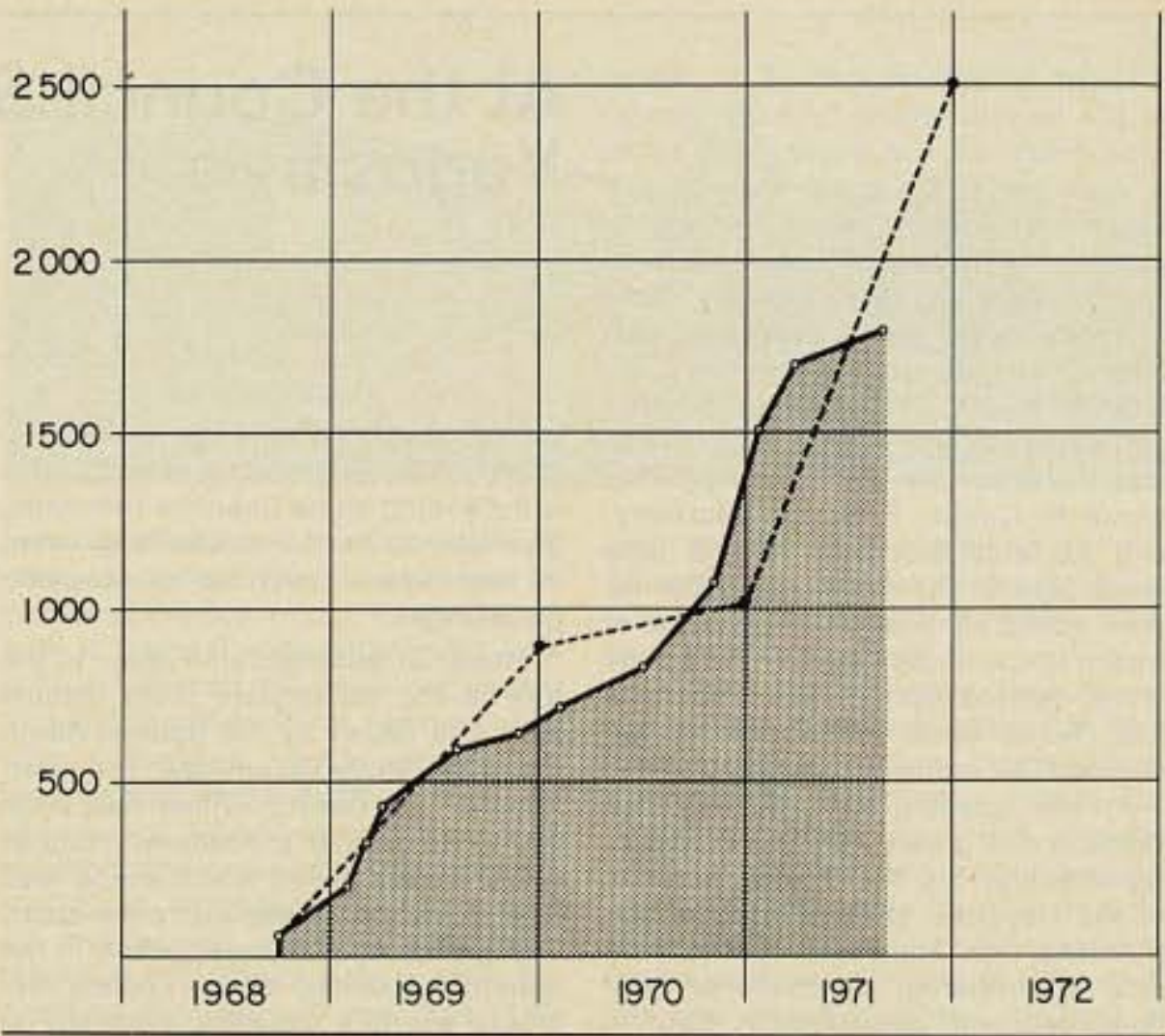

\section{Society News}

\section{Member Societies}

E. Spring, Helsinki, has been elected President of the Finnish Physical Society in succession to J. Kantele, Jyväskylä.

In the Physical Section of the Union of Czechoslovak Mathematicians and Physicists the new Chairman of the Czech Section is F. Lukes, Brno, in succession to L. Paty. Prague, and the new Chairman of the Slovak Section is J. Madar, Bratislava, in succession to L. Hrivnak, Zilina.

\section{Divisions}

The third Annual Meeting of the Quantum Electronics Division was held in Hull from 6-8 September, 1971. The topic was Laser-Plasma Interaction and the papers covered the whole range of the subject from high-power laser design to laser scattering.

The dominant theme of the session on recent advances in laser technology was $\mathrm{CO}_{2}$ lasers, with reports on developments in T.E.A., gas dynamic and c.w. laser systems and estimates of the performance of electron beam high-pressure devices. A description and some preliminary results of the 1000] Nd: glass laser built at the Lebedev Institute, Moscow, were given by N. G. Basov, and a survey of the
C.G.E. chemical laser systems completed the session.

Several groups reported the observation of neutrons associated with fusion reactions from deuterium and deuterated polythene targets irradiated by high power $\mathrm{Nd}$ : glass lasers. It was found that the neutron emission was correlated with a high target reflectivity and anomalous hard $X$-ray flux. These results were ascribed to plasma instabilities driven by the radiation which together with various studies of X-ray emission from laserproduced plasmas aroused much interest in the sessions on solid target interactions. The observation of antiStokes Raman scattering from solid hydrogen targets raised the question as to whether this process could be the origin of the initial ionization in solid-target interactions. Studies of the interaction of laser-produced plasmas with both magnetic fields and low density plasmas illustrated their use as a laboratory tool in plasma physics.

Gas breakdown studies emphasized two topics : self-focusing and $\mathrm{CO}_{2}$ laser gas breakdown. Despite a large amount of experimental data no conclusions were reached as to the origin of the self-focusing phenomena in gas breakdown. Studies of $\mathrm{CO}_{2}$ breakdown are still in a preliminary state and observations of both breakdown and expansion were presented. One of the major fields of future study in laserplasma interactions will probably be the heating of plasma by $\mathrm{CO}_{2}$ lasers. Aspects of the heating of pellets and $O$-pinches were discussed and some 